\title{
Mattieson, Christiane, Die Rationalisierung des Menschen. Architektur und Kultur der deutschen Arbeitsämter 1890-1945
}

Rachid L'Aoufir

\section{OpenEdition}

Édition électronique

URL : http://journals.openedition.org/ifha/1721

DOI : 10.4000/ifha.1721

ISSN : 2198-8943

\section{Éditeur}

IFRA - Institut franco-allemand (sciences historiques et sociales)

Référence électronique

Rachid L'Aoufir, « Mattieson, Christiane, Die Rationalisierung des Menschen. Architektur und Kultur der deutschen Arbeitsämter 1890-1945 », Revue de I'IFHA [En ligne], Date de recension, mis en ligne le 01 janvier 2008, consulté le 22 septembre 2020. URL : http://journals.openedition.org/ifha/1721 ; DOI https://doi.org/10.4000/ifha.1721

Ce document a été généré automatiquement le 22 septembre 2020.

(CIFHA 


\title{
Mattieson, Christiane, Die Rationalisierung des Menschen. Architektur und Kultur der deutschen Arbeitsämter 1890-1945
}

\author{
Rachid L'Aoufir
}

1 Cet ouvrage est consacré aux bâtiments construits pour abriter des agences pour l'emploi. Les sciences historiques ont négligé autant ces immeubles que leurs architectes. Certes, l'histoire sociale s'est intéressée aux institutions de l'intermédiation du travail, mais elle a peut-être trop insisté sur des aspects quantitatifs au détriment d'aspects qualitatifs. L'auteur propose de combler cette lacune en mettant en évidence les interactions entre les fonctions socio-économiques de ces immeubles et leur architecture extérieure et intérieure.

2 Pour cela, elle concentre son travail sur la phase de gestation des bâtiments. Ceci lui permet de montrer le rôle initiateur de la société civile dans l'émergence de l'intermédiation du travail comme un bien public qui finit par être représenté dans des immeubles spécifiques. En multipliant les points d'entrées et les perspectives, elle réussit à montrer comment des pratiques sociales souvent conflictuelles aboutissent à la création de nouvelles normes et institutions. Elle revalorise notamment le rôle du mouvement des Bourses du travail (Arbeitsnachweis-Bewegung), des associations de femmes et des syndicats. Plutôt que de présenter une thèse forte ou de chercher à vérifier une théorie, l'auteur fait confiance à sa grille de lecture qui est suffisamment complexe pour capter avec nuance les enjeux locaux, nationaux, sociaux et économiques de la structuration du marché du travail.

3 Son analyse se fonde principalement sur des archives communales. Celles-ci renferment des monographies commémoratives des Agences pour l'emploi de différentes villes, des documents de travail des assemblées communales, des rapports annuels, des statistiques, des coupures de journaux, des dossiers des offices de la construction, le tout accompagné de photographies, d'esquisses et de plans 
architecturaux. L'exploitation de la presse spécialisée et de la littérature fictive permettent de suivre la manière dont les compromis se sont dessinés dans les débats. Avec cette somme de documents, l'auteur peut rendre compte des perceptions des architectes, des décideurs dans les administrations publiques, des personnes à la recherche d'un emploi, des employés des offices pour l'emploi. L'institution se trouve ainsi insérée dans un tissu narratif dense, clairement filtré et exprimé dans un style concis.

4 L'ouvrage compte deux chapitres introductifs et quatre qui développent le thème. Le troisième offre un aperçu sur l'histoire sociale et économique de l'intermédiation du travail depuis le Moyen Âge et un bon résumé de l'ouvrage. On gagne à étudier les nombreuses photos qui parcourent le livre avant d'en poursuivre la lecture. Les chapitres suivants sont consacrés à des études de cas dans plusieurs villes selon une clef chronologique : la fin du Deuxième Reich, la République de Weimar, le Troisième Reich. Cette périodisation est justifiée par l'évolution du statut de l'intermédiation du travail, qui est de plus en plus centralisée avant de devenir un monopole d'État. Chaque chapitre présente des études de cas, douze en tout, dont Berlin, Cologne, Dresde, Hambourg, et est clos par une section qui dégage l'évolution des méthodes de mesure des travailleurs et du travail et des concepts sous-jacents aux actions.

5 En 1894, la Prusse comptait 5216 intermédiaires professionnels, 734 Bourses du travail liées à des corporations, 30 liées à des associations d'employeurs et 197 liées à des institutions communales. Les intermédiaires professionnels répondaient surtout à la demande de l'agriculture, de la restauration et des foyers privés. Cette activité était souvent liée au prêt sur caution, à l'hôtellerie et à la restauration. On retrouve ici un schéma économique en deux temps typique : l'expression non coordonnée d'un même besoin par différents corps professionnels de différentes régions ; le souhait des entrepreneurs établis d'augmenter la rentabilité de leurs circuits commerciaux en y ajoutant de nouvelles activités. Le premier mouvement donne lieu à la prise de conscience d'un problème commun dépassant les cloisonnements corporatistes et régionaux. Le second aboutit à la spécialisation puis à la différentiation fonctionnelle des nouvelles activités. Dans le courant du XIXe s., il est possible d'observer ces dynamiques aussi pour la banque et l'édition. L'État décide d'encadrer les biens publics que sont l'alimentation de l'économie en argent, en information et en travail d'abord par des concessions. Ensuite, la réglementation est affinée en fonction des expériences respectivement par l'adaptation des mesures prudentielles et de la censure. C'est la Charte industrielle de 1900 qui soumet l'intermédiation professionnelle à une obligation de concession. Cette reconnaissance publique donne lieu à des cofinancements débloqués par les communes et à la construction du premier bâtiment spécifique à Berlin.

6 La difficulté à offrir du travail aux millions de soldats démobilisés après la Première Guerre mondiale démontre toutefois l'insuffisance des mécanismes professionnels privés. Ils sont alors interdits par la loi de 1922 relative aux Bourses du travail (Arbeitsnachweisgesetz). Cette activité tombe dans le giron de l'administration publique avec la loi de 1927 qui va généraliser la dénomination d'office pour l'emploi (Arbeitsamt). Cette loi introduit également l'assurance chômage comme droit garanti par l'État, remplaçant ainsi le régime caritatif communal très inégal. L'Association des Bourses du travail allemand est dissoute pour laisser la place à un organisme de droit public, l'office de Empire pour l'intermédiation du travail et l'assurance chômage. Son 
autogestion est supprimée par l'introduction du principe du Führer en 1933. Cette centralisation homogénéise l'architecture extérieure et intérieure des bâtiments des offices pour l'emploi, une tendance qui s'était déjà dessinée dans le cadre de la rationalisation du traitement du chômage de masse.

7 L'auteur a raison d'insister sur les rapports entre le travail et la maîtrise de l'espace que reflète l'action conjuguée des acteurs privés et de l'État. La nouvelle mobilité régionale et sociale au sein de l'Empire fait passer le nombre de villes dites grandes de 8 à 48 entre 1871 et 1914. Les pouvoirs publics ont besoin de gagner une nouvelle visibilité sur ces mouvements anonymes. Les statistiques produites par diverses initiatives privées alimentent le savoir de l'administration publique qui finit par développer ses propres outils de gouvernance. La séparation des personnes à la recherche d'un emploi selon le sexe, la formation et l'âge se retrouve dans la division spatiale des immeubles. Des salles sont réservées aux femmes, des entrées aux travailleurs sans qualification. Les Bourses du travail puis les offices pour l'emploi remettent de l'ordre dans le chaos social.

L'assurance chômage institue une relation monétaire qui a pour avantage d'être facilement mesurable et de donner prise sur l'individu et sur les masses. Le découpage de l'espace intérieur des bâtiments reflète aussi les étapes de plus en plus rationalisées de l'intermédiation. Mais la représentation dans les médias des queues de chômeurs dépassant largement les limites des immeubles trahit la difficulté à maitriser l'attribution des êtres humains à des lieux spécifiques. Ce livre, qui est tiré d'une thèse de doctorat, est bien conçu. Il contient de nombreuses informations intéressantes et sait éveiller la curiosité du lecteur. On soulignera le souci de l'auteur de faire un stage dans une Agence pour l'emploi afin de mieux saisir son objet. On découvre ainsi une sensibilité et une indépendance d'esprit rafraîchissantes en dépit d'un sujet qui peut paraître rugueux à priori.

9 Rachid L'Aoufir (Berlin) 